\title{
ORGANIZACIÓN FUNCIONAL DE LAS COMUNIDADES DE MACROINVERTEBRADOS ACUÁTICOS DE UN SISTEMA FLUVIAL DE MONTAÑA (SISTEMA CENTRAL, RÍO MANZANARES, ESPAÑA)
}

\author{
A. Monzón ${ }^{1}$, C. Casado' ${ }^{1}$ C. Montes ${ }^{1}$ y D. García de Jalón ${ }^{2}$ \\ 1. Dpto. Ecología, Universidad Autónoma de Madrid. Cantoblanco, Madrid, España. \\ 2. Escuela Técnica Superior de Ingenieros de Montes, Universidad Politécnica de Madrid, 28040 Madrid, España.
}

Palabras clave: community organization, functional feeding groups, macroinvertebrates, River Continuum, streams trophic ecology, Spain.

\begin{abstract}
FUNCTIONAL ORGANIZATION OF MACROINVERTEBRATE COMMUNITIES IN A MOUNTAIN STREAM SYSTEM (CENTRAL SYSTEM, MANZANARES RIVER, SPAIN)

Relationships between the organic matter and the community functional structure of macrobenthos was analized under the conceptual frame of the River Continuum Concept (RCC).

The study was located in the upper reaches of Manzanares Basin (Central Spain) in a size range from first order streams to four order river.

Sestonic organic matter is characterized by the predominance of the dissolved fraction over the particulated one. Sestonic coarse particulate organic matter $(\mathrm{CPOM})$ generally is decreasing with stream order increasing, while the fine particulate organic matter (FPOM) increases.

El Tranco Reservoir affects downstream reaches by the decrease of the sestonic organic matter, specially of FPOM and UFPOM (ultrafine particulate organic matter).

The benthic organic matter does not follow any general pattern, being greatly controlled by local factor. Periphyton and macrophyte gross primary production and production/respiration rate neither reflect any clear tendency along the gradient, with low values of production.

The macrobenthic community trophic structure follows in a general way the patterns predicted by the RCC. When each trophic group is relating with their respective food resources, the correlation of shredders-CPOM, filterers-DOM and scrapers-P/R (Production-Respiration rate), scrapers-PB were significant. However, the correlation of collectors-F-UFPOM and predators-all others invertebrates were not significant. This show the important of food quality in this fraction (F-UFPOM) and the great fluctuation dynamic of populations.
\end{abstract}

\section{INTRODUCCIÓN}

Durante los últimos 10 años se han comenzado a configurar distintos modelos conceptuales que permitan una visión integradora de los sistemas fluviales, equivalente al gradiente o eje oligotrofia-eutrofia en los lagos (Cushing et al., 1980). De esta forma se entra en una nueva etapa de la ecología fluvial, en la que priman las investigaciones de carácter holístico sobre aquellas de tipo descriptivo (BENKE et al., 1988).

Limnetica, 7 97-112 (1991)

(C) Asociación Española de Limnología, Madrid, Spain
Se parte de una concepción discreta del río (Illes \& Botoseanu, 1963) y se deriva hacia otra de unidades continuas, que tiene su máximo exponente en el River Continuum Concept (R.C.C.) (VANNOTE et al., 1980). Inmediatamente se suscitan ávidas discusiones y comentarios (BARNES \& Minshall, 1983, Statzner \& Higler, 1985, MinsHALl et al., 1985a), encontrándonos en la actualidad en una etapa de evolución y discusión de sus premisas generales.

En este contexto y a escala mundial, se desarrollan trabajos que intentan contrastar la aplicabilidad del modelo funcional en ríos con característi- 
cas ambientales distintas de aquellas en las que se gestó esta hipótesis (CZAYA, 1983, Giudicelli et al., 1985, Barmuta, 1988, Ryder \& SCOtT, 1988) y tomando en consideración factores de carácter antrópico (WARD \& STANFORD, 1984). En muchos casos sin embargo, el empleo de parámetros meramente descriptivos ha hecho que los argumentos teóricos desarrollados no puedan ser contrastados con datos experimentales. Esta circunstancia se acentúa en el caso de la Península Ibérica, ya que es a finales de los años 70 cuando se inicia realmente el estudio limnológico de los ríos españoles.

Los trabajos desarrollados durante y a partir de este período se articulan en tres grandes líneas de actuación. En primer lugar, el mayor interés se centró en la búsqueda y adaptación de sistemas biológicos de diagnóstico de la calidad de las aguas, derivando de ello el análisis sistemático, faunístico y biogeográfico de los organismos que viven en sus aguas, prestándole especial atención al grupo de los macroinvertebrados y secundariamente a las comunidades piscícolas (GARCía DE $\mathbf{J}_{\mathrm{A}}$ Lón, 1979, 1980; PrAT, 1981; PUIG et al., 1981; PRAT et al., 1982, entre otros). En segundo lugar, tenemos estudios descriptivos de los ecosistemas fluviales centrados en el quimismo de sus aguas y en el inventario, tipificación, clasificación y cartografía de sus comunidades (GonZÁlez et al., 1983; PrAt et al., 1984; PrAT et al., 1986; PrAt et al., 1988, entre otros). Y por último, y aunque limitado en el tiempo y de un modo puntual se ha intentado abordar su estudio desde un punto de vista más funcional (GARCía DE JALÓN et al., 1988; Casado et al., 1989).

El objetivo principal de este trabajo es analizar cuantitativamente, dentro del marco conceptual del «río como un continuo», la evolución de una serie de variables funcionales a lo largo de un gradiente longitudinal de un curso fluvial del Sistema Central Ibérico.

De esta forma, se analizan variables de carácter funcional como la fracción de materia orgánica transportada y bentónica, la productividad y la densidad y biomasa de las comunidades de macroinvertebrados, contrastando los datos obtenidos con las previsiones generadas por este modelo conceptual, desde el punto de vista de su aplicabilidad a las cuencas fluviales del centro de la Península Ibérica.

\section{MATERIAL Y MÉTODOS}

\section{Área de estudio}

Previamente a la elección de la zona de estudio, se realizó un reconocimiento general de la cuenca del río Manzanares (Monzón, 1986) delimitándose una serie de sectores ambientales homogéneos respecto a características del medio físico y humano. Esto permitió considerar a la cuenca alta del eje principal de este río como el sector más apropiado para el estudio, ya que es la zona menos alterada y en la que se recoge mejor la variabilidad ambiental a lo largo del gradiente funcional de organización de las comunidades.

En total, se seleccionaron 3 estaciones de muest $\mathrm{r}$ e de orden creciente (STRAHLER, 1957) dentro de la variabilidad ambiental presente en el sector (Garganta, Maliciosa y Parque). Por otro lado, se escogieron otras tres estaciones para controlar desviaciones en el modelo, como son la temporalidad (Arroyo Mediano) y la retención artificial de agua, en un pequeño estanque («represa del Tranco») y en un embalse («Santillana») de $91 \mathrm{Hm}^{3} \mathrm{de}$ capacidad máxima de almacenamiento (estación de Navallar). Este embalse es uno de los más antiguos de la provincia (data de 1908) y está destinado principalmente al abastecimiento de Madrid. Actualmente, se encuentra en un avanzado estado de eutrofización ya que recibe un gran número de vertidos de urbanizaciones y ayuntamientos colindantes. Las sueltas de agua desde este embalse son completamente ocasionales y puntuales en el tiempo, pero de un volumen considerable (MoNTES et al., 1987).

A pesar de que el empleo de la variable morfométrica lineal «orden» como un indicador de diversas características geomorfológicas ha sido criticado por diversos investigadores (HuGHES \& OMERMIK, 1983; VIDAL-ABARCA, 1985) nosotros lo hemos escogido por su carácter sintético, facilitando en estudios de este tipo la comparación entre diferentes tamaños de ríos.

La localización geográfica de las estaciones se representa en la figura 1 y las características fisiográficas más relevantes se recogen en la tabla 1 .

El sector donde se sitúan los tramos fluviales estudiados se inserta en un paisaje abrupto con fuertes pendientes, sobre un sustrato cristalino de escaso aporte potencial de iones. Los terrenos cu- 
biertos por el embalse de Santillana hasta el tramo medio del arroyo Mediano, constituyen una mancha de materiales cuaternarios aluviales (gravas, arenas, arcillas y limos). El clima, con marcadas diferencias estacionales, es predominantemente frío, con una temperatura media anual de $8-10{ }^{\circ} \mathrm{C}$ y una vegetación adaptada a un régimen semi-húmedo (1500-800 mm de precipitación media anual). El régimen hidrológico natural, clasificado de pluvionival, no ha sido alterado en las estaciones situadas por encima del embalse de Santillana (AguiLó, 1983).

Debido a sus características paisajísticas y a la elevada demanda de zonas verdes para esparcimiento por parte de la población de Madrid, este área sufre una fuerte presión de tipo recreativo soportando una gran afluencia de público, principalmente los fines de semana y vacaciones estivales. Sin embargo, al estar incluida en el «Parque Regional de la cuenca alta del Manzanares» se ve li- bre de vertidos urbanos hasta su entrada al pueblo de Manzanares el Real.

\section{Muestreo}

Dentro de los cuatro períodos hidrológicos que en general se reconocen en los ríos del Sistema Central, este estudio se desarrolló dentro de uno de los períodos de menor caudal, en concreto el del invierno de 1986 (mediados de enero a principios de marzo), ya que ésta es la época en la que los cauces presentan una mayor estabilidad ambiental (no se producen grandes y bruscos incrementos de caudal) y sus comunidades presentan una alta organización y diversidad (CASADO, 1986) ya que no sufren las condiciones adversas del estiaje (típicas del otro período seco y estable hidrológicamente) ni de las grandes crecidas (lluvias otoñales y el deshielo de primavera).

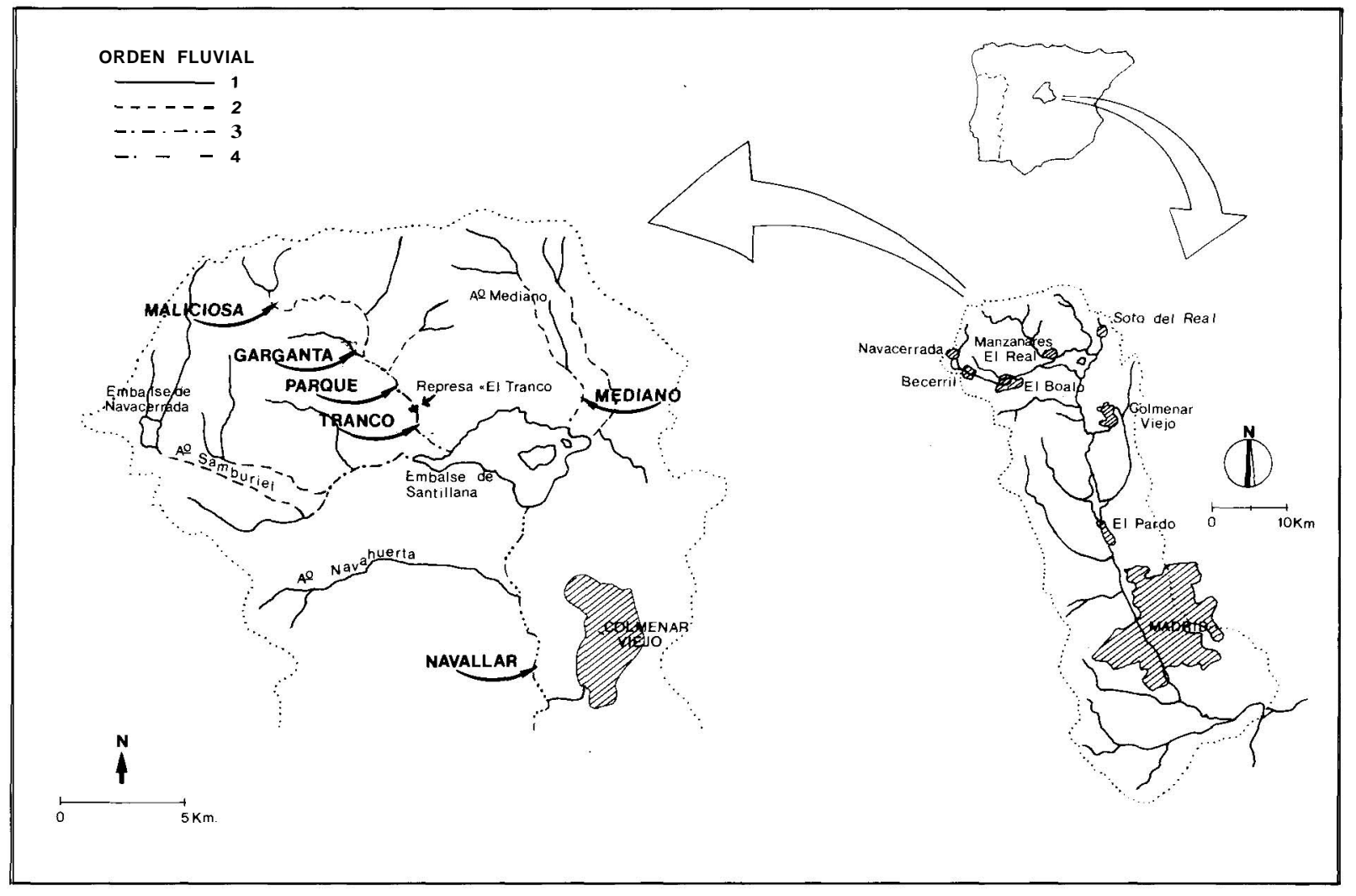

Figura 1.- Localización geográfica del río Manzanares, con la situación de las estaciones de muestreo Map of the high basin of the Manzanares river, showing sampling stations. 
Tabla 1.- Características fisiográficas más importantes de las estaciones de muestreo. a: $\mathrm{RM}=\mathrm{roca}$ madre, $\mathrm{G}=$ guijarros, $\mathrm{CR}=$ cantos rodados, $\mathrm{GR}=$ gravas, $\mathbf{A}=$ arenas. El símbolo * se emplea para diferenciar las estaciones de muestreo correspondientes a un orden dado, que presentan desviaciones en el modelo del R.C.C., frente a aquellas en las que se presupone un estado menos alterado por el hombre o relativamente «natural».

Description of the general physiographical characteristics of sampling stations.

\begin{tabular}{|c|c|c|c|c|c|c|}
\hline & \multicolumn{6}{|c|}{ Estaciones de muestreo } \\
\hline & Garganta & Maliciosa & Mediano & Parque & Tranco & Navallar \\
\hline Orden del río & 1 & 2 & $2^{*}$ & 3 & $3^{*}$ & $4^{*}$ \\
\hline Altitud (m) & 1.120 & 1.760 & 920 & 1.020 & 940 & 764 \\
\hline Área drenada $\left(\mathrm{km}^{\mathrm{z}}\right)$ & 4,6 & 6,2 & 19,0 & 35.8 & 51,7 & 329,1 \\
\hline Distancia al origen $(\mathrm{km})$ & 2,3 & 2,8 & 10,2 & 10,5 & 14,3 & 28,1 \\
\hline Pendiente $(\%)$ & 80,0 & 88,0 & 17,4 & 42,0 & 15,0 & 10.0 \\
\hline Tipo de valle & $\begin{array}{c}\text { En U } \\
\text { Tendido }\end{array}$ & $\begin{array}{c}\text { En V } \\
\text { Encajado }\end{array}$ & Tendido & $\begin{array}{c}\text { En U } \\
\text { Tendido }\end{array}$ & $\begin{array}{c}\text { En U } \\
\text { Tendido }\end{array}$ & $\begin{array}{c}\text { Intermedia V-U } \\
\text { Encajado }\end{array}$ \\
\hline Anchura media (m) & 2,95 & 8,05 & 10,1 & 4,7 & 10,2 & 10,6 \\
\hline Profundidad media $(\mathrm{cm})$ & 17,8 & 27,0 & 17,1 & 23,2 & 27.6 & 23,1 \\
\hline Caudal $\left(\mathrm{m}^{3} / \mathrm{sg}\right)$ & 0,33 & 1,18 & 0,24 & 0,51 & 1,16 & 1,33 \\
\hline Sustrato (a) & RM; GR; CR & RM; G & $\mathrm{A} ; \mathrm{CR}$ & GR; G & $\begin{array}{l}\text { A; isletas } \\
\text { emergidas }\end{array}$ & G \\
\hline Vegetación & Alta montaña & Alta montaña & Ribera & Ribera densa & Ribera & Ribera aclarada \\
\hline
\end{tabular}

En cada estación de muestreo se delimitó un espacio físico de trabajo de $50 \mathrm{~m}$ de orilla, con objeto de recoger la mayor heterogeneidad ambiental microespacial dentro de las características propias de un orden dado.

Todos los parámetros medidos, tanto de tipo descriptivo como funcional, fueron tomados en los hábitats erosionales sensu Cummins (1972).

Tanto la materia orgánica transportada o del seston (MOS) como la del lecho o béntica (MOB) fueron evaluadas en sus diferentes fracciones siguiendo la metodología descrita por HAwKINS \& SEDELl (1981) y CuMMINs et al. (1982): Materia orgánica gruesa (CPOM), superior a $1 \mathrm{~mm}$; materia orgánica fina (FPOM), de $1 \mathrm{~mm}$ a $50 \mu \mathrm{m}$, materia orgánica ultrafina (UFPOM) entre $50 \mu \mathrm{m}$ y 0,45 $\mu \mathrm{m}$ y materia orgánica disuelta (DOM) inferior a $0,45 \mathrm{ym}$.

En el muestreo de la materia orgánica del seston se empleó una red de $1 \mathrm{~mm}$ de luz de malla, localizada en la zona de máxima corriente durante una hora, para la recogida de CPOM; y una red de 50 ym, durante diez minutos, para la FPOM. La UFPOM fue estimada mediante el filtrado de 51 de agua a través de un filtro de fibra de vidrio de 0,45 ym, constituyendo la DOM la materia orgánica presente en el extracto de agua filtrada.
Todo el material recogido fue fijado con formalina y trasladado al laboratorio, donde tras su lavado se procedió al cálculo de la materia orgánica presente en cada fracción. Todos los datos son expuestos en términos de materia seca libre de cenizas (peso seco menos peso en cenizas, ps-pc) expresados en $\mathrm{mg} / \mathrm{m}^{3}$. El peso seco se obtuvo por secado en estufa a $60^{\circ} \mathrm{C}$ durante $24 \mathrm{~h}$, y el peso en cenizas incinerando en el horno a $500{ }^{\circ} \mathrm{C}$ durante $1 \mathrm{~h}$ (Lind, 1974; Cummins \& KLUG, 1979).

La materia orgánica béntica fué muestreada en los diferentes microhábitats presentes en cada estación, atendiendo a la velocidad de la corriente, vegetación presente y textura del sustrato, introduciendo un tubo de P.V.C. (6,8 cm de diámetro) de 1 a $4 \mathrm{~cm}$, en el lecho. El material recogido fué también fijado con formalina al 7-10\%. Las diferentes fracciones fueron separadas posteriormente en el laboratorio, por lavado de la muestra sobre una columna de tamices, calculándose posteriormente el volumen de materia orgánica libre de cenizas por aplicación de (ps-pc) $/ \pi r^{2} h$ viniendo expresado en $\mathrm{mg} / \mathrm{cm}^{3}$ ( $\mathrm{h}=$ longitud introduci$\mathrm{da} ; \mathrm{r}=$ radio del tubo). Se recogieron al menos tres muestras o «cores» en cada uno de los microhábitats diferenciados en cada estación de mues- 
treo, siendo el número total de muestras por estación variable entre 12 y 18 .

En la estima de producción primaria se siguió el método general de VollenweIder (1969) valorándose, in situ, los cambios en la concentración de $\mathrm{O}_{2}$ experimentados por la comunidad vegetal (previo lavado de la muestra para eliminar las poblaciones animales) confinada en pares de botellas transparentes-oscuras, tras un período de incubación inferior a $2 \mathrm{~h}$., mediante el método de Winkler. En cada estación de muestreo se colocaron al menos cuatro pares de botellas. El metabolismo de la comunidad se calculó mediante el cociente P/R. Este método no es el más adecuado en ríos, por lo que los resultados poseen tan sólo un valor comparativo en el espacio y en el tiempo (Wetzel \& LinKens, 1979).

Antes de efectuar el muestreo de las poblaciones de macrobentos fluvial, se compartimentó la zona en espacios homogéneos o hábitats efectivos (REsh, 1979) atendiendo a diversas variables ambientales como velocidad de corriente, textura del lecho y vegetación presente. Una vez delimitados los distintos tipos de hábitat se tomaron de 4 a 5 muestras al azar en cada uno de estos sectores homogéneos. Este método (muestreo estratificado) permite obtener una alta representatibilidad de la población al maximizar la variabilidad ambiental existente entre los distintos tipos de hábitat (RESH, 1979; Bass, 1982; Schwenveller \& Hellenthall, 1984; RESH \& PRICE, 1984).

Para la obtención de estimas absolutas de densidad del macrobentos se empleó un muestreador de caja que consiste en un paralelepípedo de 34 $\mathrm{cm}$ de lado y $50 \mathrm{~cm}$ de alto $\left(1156 \mathrm{~cm}^{2}\right.$ de superficie) con las dos caras laterales cerradas y dotado en la cara posterior de una red de $270 \mu \mathrm{m}$ de luz de malla y de una malla tensa de $1 \mathrm{~mm}$ de luz en la zona frontal, de forma que colocado contra corriente, el agua circula a través de él, impidiendo la entrada de organismos procedentes de deriva y la pérdida de organismos por los laterales al ser colocado firmemente ajustado al lecho mediante bandas de gomaespuma (Monzón, 1986). Los ejemplares recogidos en este espacio fueron conservados en formalina al $4 \%$, ya que es el que menos líquidos extrae de los tejidos, hasta proceder a su extracción, identificación y conteo en el laboratorio. Todos los macroinvertebrados fueron identificados al nivel taxonómico de especie con excepción de Diptera y en especial la familia Chironomidae, debido a las dificultades que presenta la determinación de las larvas. Posteriormente se procedió a la estima de la biomasa (peso seco) de cada especie expresada en $\mathrm{mg} / \mathrm{m}^{2}$ (RIGLER \& DowNING, 1984). Para ello, los organismos de cada especie fueron separados en tres clases de tamano, y secados en estufa a $60{ }^{\circ} \mathrm{C}$ durante $24 \mathrm{~h}$. Una vez obtenido el peso seco por individuo de cada clase, se realizaron los cálculos pertinentes para cada estación.

Todos los taxones fueron enmarcados dentro de un grupo funcional en relación a su especialización trófica, de acuerdo con las categorías establecidas por Cummins $(1973 ; 1979)$ y por MERRIT \& Cummins (1978) y seguidas por un gran número de investigadores:

- Desmenuzadores («shredders»): detritívoros-herbívoros; se alimentan de CPOM, de la microflora que se encuentra en el sustrato y ocasionalmente de macrófitos.

- Raspadores («scrapers»): herbívoros; se alimentan de perifiton.

- Recolectores («collectors-gatherers»): detritívoros; toman FPOM.

- Filtradores («filterers»): tipo especial de recolectores que a través del filtrado de la columna de agua retienen UFPOM y DOM

- Depredadores («predators»): se alimentan de otros organismos vivos.

Las categorías tróficas asignadas a cada uno de los taxones recolectados en este estudio se muestran en el Apéndice I.

Para caracterizar el quimismo de las aguas de las estaciones de muestreo se midieron una vez durante el estudio algunos parámetros químicos según la metodología de APHA (1985).

\section{RESULTADOS}

\section{Características físico-químicas de las aguas}

Los resultados obtenidos se exponen en la tabla 2. Se observa para el conjunto de las estaciones un cuadro físico-químico relativamente homogéneo, con aguas poco mineralizadas típicas de arroyos de cabecera no contaminados, localizados en cuencas silíceas. Los valores de conductividad, 
alcalinidad, dureza total, calcio, magnesio, cloruros y sulfatos son muy bajos, experimentando un ligero aumento en la última estación, aguas abajo del embalse de Santillana. A pesar del cual, los valores siguen siendo inferiores a los que les corresponderían por su posición topográfica dentro del marco geográfico de la red fluvial de Madrid (MONTES et al., 1987). No ocurre lo mismo con los silicatos, cuyos valores se encuentran dentro de las concentraciones normales en este tipo de aguas fluyentes.

Los valores de nutrientes son elevados para tratarse de aguas de cabecera de cursos altos del Sistema Central (CASADO et al., 1987), sin embargo, al no existir ningún vertido puntual, podemos suponer que la carga orgánica de estas aguas procederá de la descomposición de la materia orgánica alóctona procedente tanto del drenaje de la cuenca como de la vegetación de ribera. Por otro lado, esta carga orgánica supone un enriquecimiento del agua que, durante este período de estabilidad hidrológica, va a permitir un mayor desarrollo de los productores primarios y una aceleración en la fijación de la energía solar por parte del fitoplancton (BENKE et al., 1988), como reflejan los valores de clorofila «a» de la columna de agua (tabla 2).

En consecuencia, las diferencias que se detectan en la distribución espacial de las comunidades macrobentónicas, parecen estar ligadas a factores derivados de las variables fisionómicas de los cauces, más que a variaciones en el quimismo de sus aguas.

\section{Cantidad de alimento disponible: Fracciones de materia orgánica}

Este estudio nos revela (tablas 3 y 4) un sistema heterotrófico con predominio de entrada de energía alóctona, donde la fracción disuelta de MOS, constituye la principal fuente de energía, a excepción de la estación de orden $2^{*}$ (Arroyo Mediano), con un alto porcentaje de UFPOM $(98,39 \%)$ como consecuencia de su carácter temporal, que da lugar a una alta producción de macrófitos que al descomponerse constituyen la fuente principal de FPOM y UFPOM (SwAIM \& MEADER, 1958).

Las fracciones de CPOM y FPOM sólo suponen el $0,011 \%$ y el $0,013 \%$ respectivamente del total. Resultados similares han sido hallados en otros sistemas fluviales (SEDELl et al., 1978; NEw-

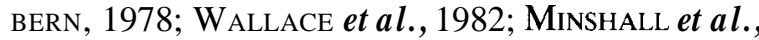
1983; GARCíA de JALÓN et al., 1988), que sin embargo, presentan rangos de variación espacial más elevados para cada una de las fracciones consideradas.

Este predominio en DOM puede derivar de la época de estudio (invierno) caracterizada por una mínima entrada de CPOM y un predominio de las

Tabla 2.- Valores registrados de algunos factores físico-químicos de la columna de agua de las estaciones seleccionadas, en invierno de 1986.

Physical and chemical characteristics of Manzanares stream water in the sampling stations.

\begin{tabular}{|c|c|c|c|c|c|c|}
\hline & \multicolumn{6}{|c|}{ Estaciones de muestreo } \\
\hline & Garganta & Maliciosa & Mediano & Parque & Tranco & Navallar \\
\hline Conductividad ( $\mu \mathrm{s} / \mathrm{cm})$ & 45,0 & 21,0 & 40,0 & 28,0 & 30,0 & 98,0 \\
\hline $\mathrm{pH}$ & 6.67 & 6,12 & 6,45 & 6,25 & 6,5 & 7,34 \\
\hline Oxígeno (mg/l) & 10,6 & 10,4 & 11,88 & 11,0 & 11,08 & 10,6 \\
\hline Alcalinidad Total (meqll) & 0,24 & 0,04 & 0,16 & 0,1 & 0,12 & 0,56 \\
\hline Cloruros (meq/l) & 0,107 & 0,084 & 0,124 & 0,078 & 0,135 & 0,208 \\
\hline Calcio (meq/l) & 0,123 & 0,047 & 0,127 & 0,079 & 0,087 & 0,475 \\
\hline Magnesio $(\mathrm{meq} / \mathrm{l})$ & 0,063 & 0,036 & 0,068 & 0,040 & 0,047 & 0,155 \\
\hline Dureza total $\left(\mathrm{mg} \mathrm{CaCO}_{3} / \mathrm{l}\right)$ & 9,4 & 4,2 & 9,8 & 6,0 & 6,8 & 31,6 \\
\hline Sulfatos (meq/l) & 0,72 & 0,72 & 2,14 & 1,43 & 3,86 & 8,14 \\
\hline Silicatos $(\mu \mathrm{g}-$ at $\mathrm{Si} / \mathrm{l})$ & 5,39 & 3,50 & 35,94 & 6,04 & 18,45 & 36,86 \\
\hline Nitrato $\left(\mu \mathrm{g}\right.$ - at $\left.\mathrm{N}-\mathrm{NO}_{3} / \mathrm{l}\right)$ & 1.64 & 10,42 & 2,74 & 0,26 & 6.82 & 10,3 \\
\hline Nitrito $\left(\mu \mathrm{g}\right.$ - at $\left.\mathrm{N}-\mathrm{NO}_{2} / \mathrm{l}\right)$ & 1,52 & 0,43 & 0,13 & 0,00 & 0,13 & 3,65 \\
\hline Fosfatos (pg- at $\left.\mathrm{P}^{-\mathrm{PO}_{4}} / 1\right)$ & 0,32 & 0.08 & 0,8 & 0,12 & 0,16 & 0,28 \\
\hline Clorofila «a» $\left(\mathrm{mg} / \mathrm{m}^{3}\right)$ & 0,42 & 0,7 & 1,39 & 2,08 & 0,7 & 0,98 \\
\hline
\end{tabular}


Tabla 3.- Cantidad media de materia orgánica transportada durante el invierno de 1986 para las distintas fracciones de material orgánica del seston, a lo largo de un gradiente fluvial de orden creciente. El signo * indica los segmentos fluviales que presentan algún tipo de intervención humana.

Longitudinal shifts in the amount of transport organic matter for the different detritus size fraction, along de Manzanares stream.

\begin{tabular}{|c|c|c|c|c|c|c|c|c|c|c|c|c|}
\hline & \multicolumn{2}{|c|}{$\begin{array}{l}\text { Garganta } \\
\text { (orden I) }\end{array}$} & \multicolumn{2}{|c|}{$\begin{array}{l}\text { Maliciosa } \\
\text { (orden 2) }\end{array}$} & \multicolumn{2}{|c|}{$\begin{array}{c}\text { Mediano } \\
\left(\text { orden } 2^{*}\right)\end{array}$} & \multicolumn{2}{|c|}{$\begin{array}{c}\text { Parque } \\
\text { (orden 3) }\end{array}$} & \multicolumn{2}{|c|}{$\begin{array}{c}\text { Tranco } \\
(\text { orden } 3 *)\end{array}$} & \multicolumn{2}{|c|}{$\begin{array}{c}\text { Navallar } \\
\left(\text { orden } 4^{*}\right)\end{array}$} \\
\hline & $m g / m^{3}$ & $\%$ & $m g / m^{3}$ & $\%$ & $m g / m^{3}$ & $\%$ & $m g / m^{3}$ & $\%$ & $m g / m^{3}$ & $\%$ & $m g / m^{3}$ & $\%$ \\
\hline СРOM & 10.14 & 0,065 & 14.77 & 0.3 & 10,454 & 0,004 & 2,28 & 0,046 & 2,07 & 0,009 & 0,73 & 0,004 \\
\hline FPOM & 2.47 & 0,016 & 0,62 & 0,01 & - & - & 13,4 & 0,027 & 2,72 & 0,013 & 0,55 & 0,003 \\
\hline UFPOM & 500 & 3.20 & 620 & 12,60 & 255.366 & 98.39 & 740 & 1,47 & 580 & 2,72 & 1.380 & 7.75 \\
\hline DOM & 15.000 & 96,70 & 4.285 & 87,1 & 4.160 & 1,6 & 49.280 & 98,48 & 20.714 & 97.25 & 16.428 & 92.24 \\
\hline TOTAL & $15.512,61$ & & $4.920,39$ & & 259536,454 & & $50.035,68$ & & $21.298,79$ & & 17.809 .28 & \\
\hline
\end{tabular}

precipitaciones en forma de nieve, con intervalos de deshielo (Robinson \& Minshall, 1986). Esto origina un mayor aporte de elementos finos no conservativos (EsCARRÉ et al., 1984) en relación a otras épocas del año.

Respecto a la evolución de la materia orgánica en el orden fluvial creciente, se observa que la DOM no presenta ninguna tendencia de variación longitudinal en el gradiente, respondiendo más a características fisiográficas locales que darán lugar a una menor o mayor persistencia de estas precipitaciones y por tanto a la lixiviación de los materiales.

En relación al resto de fracciones de MOS, se observa (tabla 3) que la CPOM presenta una tendencia general de disminución en el orden creciente, con excepción de los dos primeros órdenes debido a peculiaridades fisionómicas propias asociadas fundamentalmente al tipo de valle. Así, la estación de orden 1 está localizada en un valle ancho y tendido que facilita la entrada de materia orgánica procesada en lugares más alejados (más FPOM) por la acción combinada del viento y las precipitaciones; mientras que la estación de orden
2 está situada en un valle encajado (más CPOM) donde la materia orgánica entra casi directamente al sistema sin apenas ser procesada en el medio circundante.

La fracción de UFPOM presenta un aumento hacia la desembocadura en concordancia con el modelo del R.C.C., excepto aguas abajo de la represa del Tranco donde se observa una disminución de sus valores (tabla 3).

Las características locales condicionan también la distribución longitudinal de la MOB (tabla 4), que no responde a las predicciones de la hipótesis del R.C.C. Las estaciones con menos cantidad de MOB fueron las de orden 2 y $4^{*}$ (Maliciosa y Navallar). En la primera, el flujo turbulento anula la capacidad de retención de este tramo, movilizando los sedimentos. La segunda se encuentra en un período de lavado del lecho debido a las sueltas de agua efectuadas desde el embalse de Santillana, al verse rebasada su capacidad de almacenamiento. Es decir, el incremento de caudal origina una perturbación en el sistema, ya que altera los flujos de sedimentos y por tanto la cantidad de materia orgánica béntica. A pesar de que la inten-

Tabla 4.- Valores medios de las diferentes fracciones de materia orgánica bentónica estimadas en el invierno de 1986 a lo largo de un gradiente fluvial de orden creciente.

Longitudinal shifts in the amount of benthic organic matter for the different detritus size fractions along of Manzanares stream.

\begin{tabular}{|c|c|c|c|c|c|c|c|c|c|c|c|c|}
\hline & \multicolumn{2}{|c|}{$\begin{array}{l}\text { Garganta } \\
\text { (orden 1) }\end{array}$} & \multicolumn{2}{|c|}{$\begin{array}{l}\text { Maliciosa } \\
\text { (orden 2) }\end{array}$} & \multicolumn{2}{|c|}{$\begin{array}{c}\text { Mediano } \\
(\operatorname{orden} 2 *)\end{array}$} & \multicolumn{2}{|c|}{$\begin{array}{c}\text { Parque } \\
\text { (orden 3) }\end{array}$} & \multicolumn{2}{|c|}{$\begin{array}{l}\text { El Tranco } \\
\text { (orden 3*) }\end{array}$} & \multicolumn{2}{|c|}{$\begin{array}{l}\text { Navallar } \\
\text { (orden } 4^{*} \text { ) }\end{array}$} \\
\hline & $\mathrm{mg} / \mathrm{cm}^{3}$ & $\%$ & $\mathrm{mg} / \mathrm{cm}^{3}$ & $\%$ & $\mathrm{mg} / \mathrm{cm}^{3}$ & $\%$ & $\mathrm{mg} / \mathrm{cm}^{3}$ & $\%$ & $\mathrm{mg} / \mathrm{cm}^{3}$ & $\%$ & $\mathrm{mg} / \mathrm{cm}^{3}$ & $\%$ \\
\hline СРОМ & 8.67 & 36,52 & 0 & 0 & 8,38 & 41,43 & 4,69 & 55,77 & 7.96 & 89.53 & 0 & 0 \\
\hline FPOM & 11,49 & 48,39 & 1,64 & 66,8 & 7,56 & 37,39 & 2,37 & 28.25 & 0,93 & 10.46 & 1,04 & 63,29 \\
\hline UFPOM & 3,58 & 15,10 & 0,81 & 33,06 & 4,28 & 21,15 & 1.34 & 15,95 & 0,098 & 9,10 & 0,60 & 36,79 \\
\hline
\end{tabular}


sidad y predecibilidad de ambas perturbaciones es muy distinta (la de Maliciosa se debe a las variaciones estacionales naturales, mientras que la de Navallar es completamente artificial e impredecible), sus efectos sobre la materia orgánica béntica son muy similares, presentando ambas estaciones unos valores mínimos de FPOM y UFPOM, y nulos de CPOM. Así, la geomorfología y el régimen hidrológico constituyen dos de los principales factores de perturbación en sistemas fluviales (RESH et al., 1988). Por el contrario, las estaciones de orden 1 y $2^{*}$ (Garganta y Mediano) son las que presentan mayores concentraciones de MOB al tiempo que presentan una mayor cobertura de macrófitos, del $12 \%$ y $46 \%$ respectivamente, discurriendo ambas por valles anchos.

La presencia de embalses y pequeñas represas produce desviaciones en el modelo (R.C.C.). La existencia de la represa del Tranco entre dos localidades del mismo orden (3) provoca disminuciones en las cantidades de MOS y MOB en todas sus fracciones, actuando así a modo de pequeño sumidero. Si examinamos la razón CPOM/F-UFPOM de MOS (tabla 3) vemos que experimenta un pequeño aumento en concordancia con el comportamiento de esta represa, que retiene fundamentalmente las fracciones de F-UFPOM y en menor medida a la CPOM, ya que esta última se transporta poco y suele quedarse y consumirse en el mismo lugar de origen (HaLl, 1972). Este aumento contradice la tendencia esperada en el modelo de «Serial Discontinuity» planteado por W ARd \& STANFord (1984) para ríos regulados. Por el contrario, en la estación de Navallar, aguas abajo del embalse de Santillana, se observa una fuerte disminución de las fracciones orgánicas de mayor tamaño (tanto sestónicas como bénticas), evolucionando según el modelo del R.C.C. y el «Serial Discontinuity» ya mencionado.

En relación al metabolismo de la comunidad (tabla 5) no existe una tendencia clara de aumento o disminución en el gradiente, tanto para la producción bruta como para $\mathrm{P} / \mathrm{R}$, encontrándonos con producciones muy bajas en comparación con otros estudios (Hawkins \& SEDELL, 1981; PRINGER et al., 1988). La estación que presenta valores más altos es la de Arroyo Mediano $\left(0,12 \mathrm{mg} \mathrm{O}_{2} / \mathrm{h}\right)$ de carácter temporal con una gran producción de macrófitos que procederán a descomponerse de forma eficiente cuando se seque el lecho, consti-
Tabla 5.- Valores medidos de PB y P/R para el conjunto de los productores primarios a lo largo de un gradiente fluvial de orden creciente.

Longitudinal shifts in the gross production and production-respiration rate along the sampling stations of the Manzanares strearn.

\begin{tabular}{lccccc}
\hline \hline & $\begin{array}{c}\text { Garganta } \\
(\text { orden 1) }\end{array}$ & $\begin{array}{c}\text { Maliciosa } \\
(\text { orden 2) }\end{array}$ & $\begin{array}{c}\text { Mediano } \\
(\text { orden 2*) }\end{array}$ & $\begin{array}{c}\text { El Tranco } \\
(\text { orden 3*) }\end{array}$ & $\begin{array}{c}\text { Navallar } \\
(\text { orden 4*) }\end{array}$ \\
\hline \hline PB $\left(\mathrm{mgO}_{2}\right.$ h) & 0,029 & 0,0017 & 0.12 & 0,0049 & 0.034 \\
PIR & 0,017 & $9,84 \cdot 10^{-4}$ & 0,033 & 0,0082 & 0,0043 \\
\hline
\end{tabular}

tuyendo así la fuente principal de F-UFPOM cuando vuelva a circular el agua (Swain \& MEADER, 1958). A esta estación le sigue la de Navallar $\left(0,034 \mathrm{mg} \mathrm{O}_{2} / \mathrm{h}\right)$ pero con una comunidad de productores primarios constituida por perifiton. La estación de Garganta, (orden 1) posee valores más elevados $\left(0,029 \mathrm{mg} \mathrm{O}_{2} / \mathrm{h}\right)$ a los esperados en relación a su posición en el gradiente, debido a su localización en un valle tendido (ya apuntado en relación a la CPOM) con vegetación de alta montaña (piornal). Esta desviación del modelo del R.C.C., donde se predicen valores de productividad bajos o menores respecto a órdenes crecientes, con predominio de entrada de energía alóctona al sistema en arroyos de primer orden, es ya recogida por VANNOTE $\boldsymbol{e}$ t al. (1980). También ha sido recogido, aunque con posterioridad (GIUDICELLi $\boldsymbol{e}$ a $\boldsymbol{l} .$, , 1985) el funcionamiento excepcional de los ríos temporales.

\section{Estructura funcional: grupos tróficos}

La evolución predecible de la estructura trófica de la comunidad según el orden fluvial creciente, es que los desmenuzadores sean mayoritarios, junto con los recolectores en las zonas de cabecera, para después disminuir rápidamente, al tiempo que se espera un incremento de los filtradores en los órdenes mayores; los raspadores deben coevolucionar con la producción primaria del perifiton, dominando en los tramos medios; mientras que los depredadores se muestran más o menos constantes en el gradiente.

La tabla 6 recoge la estructura trófica de la comunidad del macrobentos en términos de densidad y biomasa. La contribución relativa de cada grupo funcional a la organización de la comunidad se ha determinado examinando los cocientes 
Tabla 6.- Estructura trófica de las comunidades bentónicas expresada en términos de densidad (individuos $\left./ \mathrm{m}^{2}\right)$ y biomasa ( $\mathrm{mglm}{ }^{2}$ ) a lo largo de un gradiente fluvial de orden creciente.

Longitudinal shifts in the density and biomass of different functional feeding group along Manzanares stream.

\begin{tabular}{|c|c|c|c|c|c|c|c|c|c|c|c|c|}
\hline & \multicolumn{2}{|c|}{$\begin{array}{r}\text { Garganta } \\
\text { (orden I) }\end{array}$} & \multicolumn{2}{|c|}{$\begin{array}{r}\text { Maliciosa } \\
\text { (orden 2) }\end{array}$} & \multicolumn{2}{|c|}{$\begin{array}{c}\text { Mediano } \\
\left(\operatorname{orden} 2^{*}\right)\end{array}$} & \multicolumn{2}{|c|}{$\begin{array}{c}\text { Parque } \\
\text { (orden 3) }\end{array}$} & \multicolumn{2}{|c|}{$\begin{array}{c}\text { Tranco } \\
\left(\operatorname{orden} 3^{*}\right)\end{array}$} & \multicolumn{2}{|c|}{$\begin{array}{c}\text { Navallar } \\
\text { (orden } 4^{*} \text { ) }\end{array}$} \\
\hline & ind $/ m^{2}$ & maim $^{2}$ & ind $/ m^{2}$ & $m g / m^{2}$ & $i n d / m^{2}$ & $m g / m^{2}$ & indim $^{2}$ & $m g / m^{2}$ & $i n d / m^{2}$ & $m g / m^{2}$ & ind $/ m^{2}$ & $m g / m^{2}$ \\
\hline Desmenuzadores & 69.21 & 104,1 & 819,21 & 356,09 & 395,33 & 483,05 & 205,88 & 93.85 & 67,47 & 137,33 & 12,97 & 5.27 \\
\hline Raspadores & 365,91 & 365,64 & 62,33 & 39,32 & 484,42 & 396,1 & 354,67 & 40,152 & 70,93 & 16,51 & 17,30 & 11,5 \\
\hline Recolectores & $1.219,02$ & 447,29 & $1.068,34$ & 349,91 & $1.351,64$ & 655,6 & $2.058,81$ & 650.64 & $1.605,53$ & 388,75 & 499,57 & 101.23 \\
\hline Filtradores & $1.022,49$ & 518,38 & 278,54 & 683,2 & 462.80 & 516,8 & $9.844,29$ & $2.224,5$ & $1.465,39$ & 386,6 & 10,8 & 53 \\
\hline Depredadores & 58,82 & 217.84 & 224,47 & 355,47 & 482,27 & 437,86 & 160,9 & 266,59 & 145.32 & 262,82 & 16.86 & 15.67 \\
\hline TOTAL & $2.735,45$ & $1.653,25$ & $2.452,89$ & $1.783,99$ & $3.176,46$ & $2.489,41$ & $12.624,55$ & $3.275,73$ & $3.354,64$ & 1.192 .01 & 557,5 & 187,67 \\
\hline
\end{tabular}

de dominancia (densidad o biomasa de un grupo funcional/densidad o biomasa total de invertebrados) que se representan en las figuras 2 y 3 . Se observa que los colectores (recolectores-filtradores) constituyen el grupo dominante con un aporte numérico del $83,87 \%$ a la densidad de la comunidad, representando el $65,93 \%$ de la biomasa total, en respuesta al recurso prioritario de materia orgánica del seston, en sus fracciones fina, ultrafina y disuelta.

Como puede observarse en las figuras 2 y $\mathbf{3}$, en la estación de orden 3 (Parque) se produce un incremento grande de los filtradores (con altas densidades de Simuliidae debido sobre todo al flujo turbulento del agua), en detrimento de los recolectores, como respuesta a la práctica inexistencia de productores primarios, entre ellos macrófitos, principal fuente de partículas finas de alta calidad (Minshall et al., 1983). Esta relación se invierte en el orden 4 (Navallar), con una comunidad dominada por los recolectores. Las bajas densidades de individuos encontrados en esta última son consecuencia inmediata de las elevadas sueltas efectuadas desde el embalse de Santillana.

Los desmenuzadores y raspadores muestran una tendencia decreciente al aumentar el orden del río. Los desmenuzadores constituyen una fracción mayor en la estación de orden 2 (Maliciosa) frente a la de orden 1 (Garganta), donde adquieren mayor importancia los raspadores. Esto es lógico si recordamos que el sistema se inicia en una zona de pastizal-piornal de alta montaña y por tanto con una mayor intensidad luminica.

Los depredadores se mantienen más o menos constantes a lo largo del gradiente, aumentando li- geramente en las estaciones de orden 2 (Maliciosa y Arroyo Mediano).

En la estación de orden $2^{*}$ (Mediano) la contribución relativa de los distintos grupos funcionales está muy equilibrada, aunque con el predominio general de los recolectores. Su carácter temporal unido a las características fisionómicas propias (valle tendido, pendiente suave, tamaño medio de las partículas del sustrato, etc.), parecen propiciar una mayor heterogeneidad ambiental que favorecería esta situación. En este sentido, podríamos contemplar la temporalidad como un factor de perturbación, no demasiado fuerte ni extremo, que va a permitir una mayor diversificación ambiental y de entradas de nutrientes, que conducirá a una estructura funcional más equilibrada y a un aumento de la diversidad biótica, de acuerdo con la hipótesis de la perturbación media (WARd \& StAnFord, 1983).

Los filtradores disminuyen en la estación situada aguas abajo de la represa del Tranco, como si la regulación llevara a un rejuvenecimiento del sistema, de acuerdo con lo anteriormente expuesto para la materia orgánica. Este descenso también se observa en la estación de Navallar, aguas abajo de Santillana, aunque unido a un ligero incremento de los raspadores, como cabría esperar en función de los valores de P.B.

\section{Relación entre los distintos grupos tróficos y el alimento disponible}

De acuerdo con el modelo del río como un continuo, la distribución de los macroinvertebrados acuáticos reflejará los cambios en la cantidad y ti- 
pos de alimento disponibles, lo que varía con el orden del río, sin olvidar que existen ciertas limitaciones a estas premisas. En general, la organización de las comunidades de macrobentos fluvial puede ser explicada en base al estado medio de las variables ambientales y su grado de variabilidad temporal y heterogeneidad espacial (MINSHALL $\boldsymbol{e t}$ al., 1985 b). Estas comunidades se estructuran a lo largo del gradiente, de tal forma que las comunidades de los tramos inferiores capitalizan la ineficiencia del procesado de materia orgánica de los tramos superiores. En este sentido, se analizan las relaciones entre los distintos grupos tróficos y las diferentes fuentes alimenticias, a través del cálculo del coeficiente de correlación entre las distintas fracciones orgánicas y la densidad y biomasa de los grupos funcionales: desmenuzadores con CPOM; recolectores con F-UFPOM; filtradores con DOM; raspadores con la PB y P/R y los depredadores con las presas.

Los desmenuzadores muestran correlaciones significativas, en términos de densidad y biomasa con los niveles de CPOM del seston (densidad: $\mathrm{r}=.50, \mathrm{p}<.01, \mathrm{n}=27$; biomasa: $\mathrm{r}=.51$, $\mathrm{p}<.01, \mathrm{n}=27)$. Ya que en este estudio sólo se analizó la cantidad de recursos y no la calidad, esta correlación significativa implicaría que o la calidad no es tan importante como se ha reseñado en otros trabajos (ANDERson \& CuMMINs, 1979; ANDERSON \& SEDELL, 1979) o que dentro de esta CPOM se encuentran fracciones homogéneas de alta calidad.

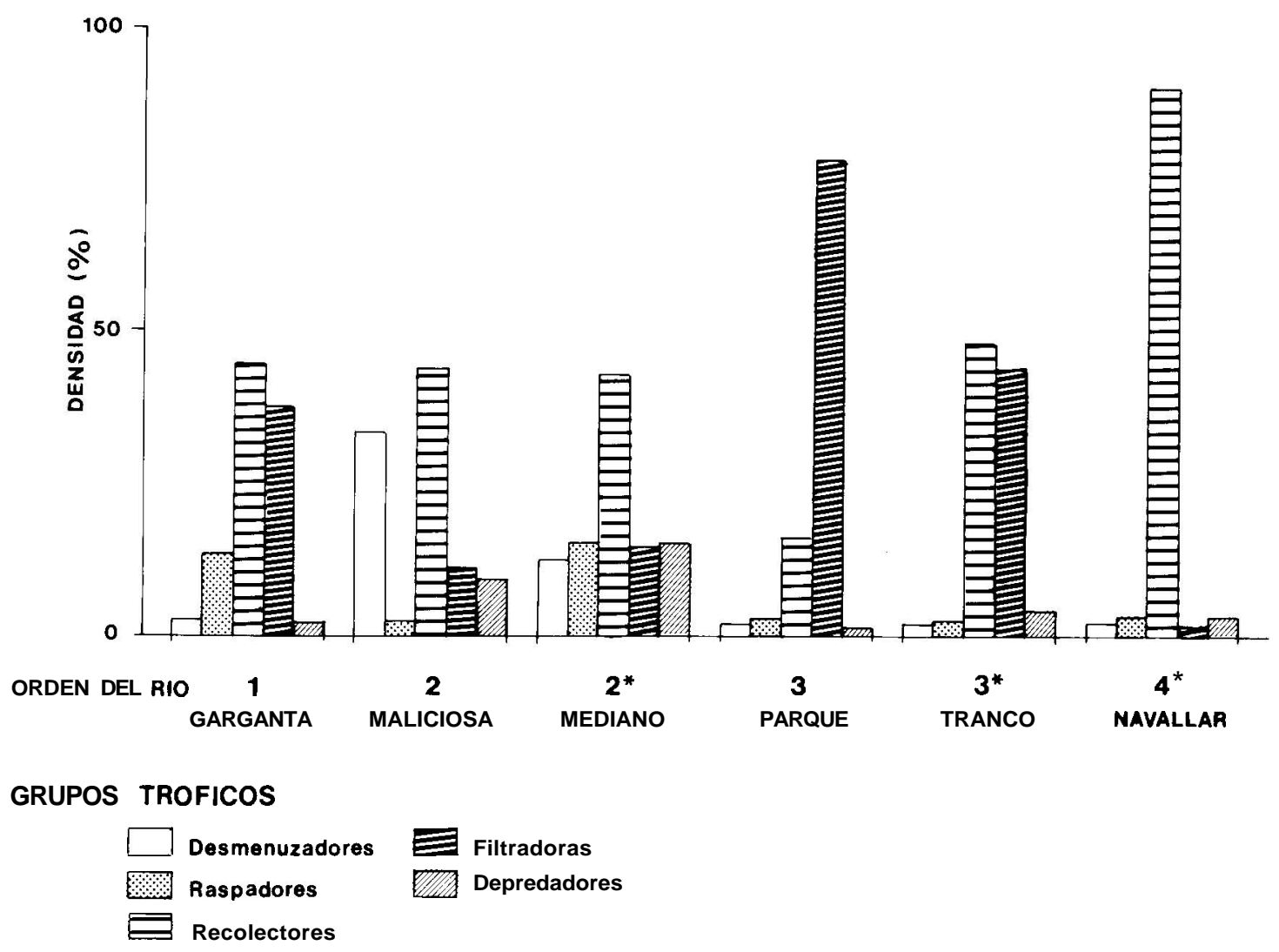

Figura 2.- Estructura trófica de las coniunidades bentónicas expresada en porcentaje de su densidad para cada una de las estaciones muestreadas. El símbolo * indica los órdenes fluviales que presentan algún tipo de intervención humana.

Macrobenthic community trophic structure, in relative density terms, from the sarnpling stations of the Manzanares river. The syrnbol * shows the stream order which are affected by human intervention. 
Los recolectores no mostraron correlaciones significativas con la F-UFPOM del seston (densidad, $\mathrm{r}=-.13, \mathrm{n}=27$; biomasa, $\mathrm{r}=.26$, $\mathbf{n}=27)$. Una posible explicación está en que ambas fracciones están compuestas de partículas orgánicas de muy distinta naturaleza, que difieren ampliamente en sus valores nutricionales. Si tanto la FPOM como la UFPOM están compuestas de partículas de bajo valor alimenticio, relativamente grandes diferencias en cantidad, sólo reflejarán pequeñas diferencias en el verdadero alimento disponible. Para definir el valor nutritivo de esta fracción particulada deberían ser analizados parámetros cualitativos tales como la clorofila «a»/gr de F-UFPOM, así como la proporción de colonias vivas de bacterias, diatomeas y microal- gas agregadas a este tipo de fracción orgánica. Así pues, se desprende que las medidas de cantidad de F-UFPOM son inadecuadas para predecir la abundancia de los recolectores en el gradiente (Hawkins \& Sedell, 1981). Por tanto, debería prestarse más atención al conjunto mayoritario del sistema, esto es: fracción fina y ultrafina y organismos recolectores-filtradores, que por otro lado son los que plantean mayores problemas de identificación (principalmente algunas familias de dípteros), siendo muchas veces obviados en estudios de esta índole. La mayoría de los recolectores son generalistas y omnívoros selectivos (Cummins $\boldsymbol{e t}$ al., 1979) alimentándose proporcionalmente a la cantidad de alimentos disponibles dentro de ciertas preferencias, que estarán en relación con la es-

100<smiles>C1CCCCC1</smiles>

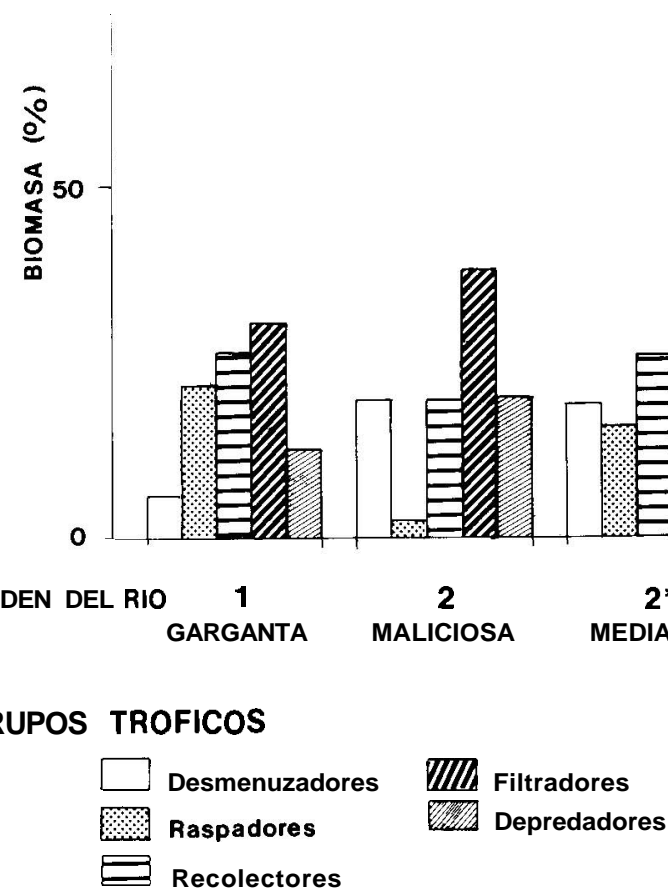

Figura 3.- Estructura trófica de las comunidades bentónicas, expresada en porcentaje de su biomasa para cada una de las estaciones muestreadas.

Macrobenthic community trophic structure, in relative biomass terms, from the sampling stations of Manzanares river. 
pecialización de sus estructuras de captura, pudiendo cambiar a lo largo del año con el estado de desarrollo del organismo. Así pues, se reconoce que correlaciones no significativas puedan ser debidas a una incorrecta clasificación de los organismos en uno u otro nivel trófico.

Los filtradores mostraron fuertes correlaciones significativas con la DOM tanto en términos de densidad como de biomasa (densidad, $\mathbf{r}=.73$, $\mathrm{p}<.01, \mathrm{n}=27$; biomasa, $\mathrm{r}=.58, \mathrm{p}<.01$, $\mathbf{n}=27)$. La fracción de DOM es importante sobre todo en invierno, cuando la producción primaria es baja, convirtiéndose así en la principal fuente de materia orgánica.

Los raspadores muestran correlaciones significativas tanto con P/R como con P.B. cuando la relación se establece en términos de biomasa $\left(\mathrm{r}_{\mathrm{P} / \mathrm{R}}=.49, \mathrm{p}<.01, \mathrm{n}=27 ; \mathrm{r}_{\mathrm{PB}}=.38, \mathrm{p}<.05\right.$, $\mathrm{n}=27$ ), pero no fueron significativos en ningún caso en términos de densidad.

Los depredadores y las presas, no muestran correlaciones significativas (densidad, $\mathrm{r}=.02 \mathrm{y}$ biomasa, $r=.07$ ). Esto puede ser debido a la propia dinámica del modelo depredador-presa, de tal forma que nos encontramos en un momento de desequilibrio dinámico entre ambas poblaciones. En este estudio las presas superaban con mucho, tanto numéricamente como en biomasa, a los depredadores.

Al correlacionar las distintas fracciones de materia orgánica béntica con los respectivos grupos tróficos, los valores obtenidos no han sido significativos en ningún caso. Este hecho puede ser debido a las bajas cantidades de materia orgánica encontrada en el lecho fluvial, como consecuencia del período en que se ha realizado el muestreo: con mínimas entradas de materia orgánica y, posterior a una época de máxima avenida (otoño) con disminución de los mecanismos de retención y exportación de los materiales hacia los tramos inferiores. Sin embargo, los mismos resultados han sido encontrados en estudios realizados en otros ríos de latitudes templadas (BARMUTA, 1988), no siendo significativas las correlaciones entre los grupos tróficos presentes y la materia orgánica béntica en ninguna época del año. Este autor sugiere que la calidad de cada una de las fracciones particuladas bénticas sería una variable predictiva más adecuada que la cantidad de las mismas.

\section{DISCUSIÓN}

A través de los resultados obtenidos, se siente la necesidad de analizar no sólo la cantidad de alimento disponible en sus diferentes fracciones sino también la calidad de éstos, ya que a la vista de los resultados, las relaciones no son tan simples como era de esperar según el modelo del R.C.C. Además sería necesario un seguimiento de todo el ciclo anual, para observar la variabilidad estacional.

En líneas generales, en este estudio se cumplen las predicciones del río como un continuo. El grupo funcional más numeroso es el de los recolectores-filtradores como respuesta al recurso mayoritario, FPOM y DOM. Tanto estos niveles tróficos como el resto evolucionan según el modelo, aumentando los primeros hacia órdenes crecientes y disminuyendo el resto.

Las mayores alteraciones están referidas a los raspadores junto con la $\mathrm{PB}$ y la relación $\mathrm{P} / \mathrm{R}$ debido, por un lado, a que el sistema se inicia en una zona de pastizal de alta montaña y, por otro, a las alteraciones introducidas por el hombre en tramos inferiores, al sustituir la vegetación natural de la cuenca por repoblaciones de Pinus sylves tris, por lo que los raspadores, dentro de su relativa importancia, son más dominantes en el tramo de orden 1.

Por otro lado, la retención del agua en la represa del Tranco, produce un empobrecimiento en materia orgánica, ya que esta represa actúa como un sumidero reteniendo principalmente las fracciones de UFPOM y FPOM y permitiendo el aclarado del agua. Una disrupción más importante es la originada por el embalse de Santillana, debido a la suelta de agua y lavado del lecho fluvial, que puede ser interpretado como una perturbación imprevisible y catastrófica del sistema, suponiendo una fuerte disrupción del mismo. En este sentido, la hipótesis dada por WARD \& STANFord (1983) «Intermediate disturbance hypothesis» y desarrollada posteriormente por RoBInSON \& MinshaLL (1986) y Risir et al. (1988) puede resultar de gran utilidad para explicar no sólo la estructura (PRAT et al., 1984) sino también la organización funcional de las comunidades fluviales.

También se observan diferencias de funcionamiento entre sectores de la red con mayor o menor permanencia del agua. Así la estación de or- 
den $2^{*}$ (Arroyo Mediano), de carácter temporal, presenta mayores concentraciones de materia orgánica particulada fina y ultrafina, posee una mayor producción bruta y diversificación de microhábitats, lo que permite un ajuste mejor entre los grupos funcionales, con una proporción importante de depredadores. El funcionamiento de los ríos temporales constituirá otra excepción al modelo (GiUdicElli et $\boldsymbol{a l}$., 1985), a pesar de lo cual podemos recurrir al R.C.C. como marco de referencia teórico para explicar las desviaciones funcionales de estos cursos de agua no permanente.

Las desviaciones del modelo son debidas fundamentalmente a características locales del sistema y podemos considerar que el modelo se cumple o no según la escala de trabajo que estemos considerando. A una escala general, en extensiones suficientemente grandes, el modelo resulta de gran utilidad, aunque si afinamos espacialmente, a una escala de mayor detalle, podemos encontrar fuertes desviaciones de sus predicciones y premisas, originadas por condiciones naturales extremas o en situaciones de cambios rápidos en espacios reducidos, que motivan la disrupción, distorsión o discontinuidad de las variables consideradas en el modelo.

La hipótesis del río como un continuo, a pesar de las excepciones geográficas o locales y presupuestos particulares con relación a las alteraciones de los sistemas fluviales, resulta de gran utilidad en el sentido de que aúna en sí muchos aspectos ya considerados con anterioridad a su formulación, que a modo de mosaico conforman el sistema hidrológico. En este sentido, el R.C.C., sirve para entender el funcionamiento a una escala general de interpretación, a partir de la cual poder encontrar otras vías de entendimiento de los procesos particulares que están ocurriendo localmente en el sistema fluvial, y que debido a su gran complejidad hacen imposible la modelización global u holística a escala muy fina de detalle. Es decir, a partir de este modelo holístico y general, podemos teorizar y construir hipótesis que modelicen el comportamiento parcial de pequeños tramos fluviales o cuadros ambientales que por distintas causas se aparten del funcionamiento general caracterizando asimismo los factores responsables de estas desviaciones.

En este sentido, el mayor interés del R.C.C. reside en que se ha constituido como una teoría o hipótesis sintética que nos permite entender desde un punto de vista holístico, los procesos ecológicos que están ocurriendo en un sistema fluvial. Ya que se trata de un concepto unificador, que engloba e interrelaciona diversos aspectos funcionales considerados con anterioridad de forma más o menos aislada, proporciona un sólido armazón teórico, que puede servir como marco de referencia al que acoplar otras hipótesis más parciales o menos globalizadoras como «The intermediate disturbance hypothesis» o «The serial discontinuity concept».

El río es un sistema monstruo (MARGaleF, 1984) por su carácter abierto y asimétrico (globalizador de las características de toda la cuenca y con múltiples gradientes, cabecera-desembocadura, centro-orilla, etc.) que dependiendo de la escala a la que sea observado nos descubre un mundo nuevo constituido por un mosaico de condiciones ambientales tales que podemos seguir descomponiendo en otras unidades menores en función de la enorme variabilidad ambiental. En último término, cada río se comporta como una unidad individual a partir de la cual pueden hallarse semejanzas o disimilitudes geográficas con otros ríos. Por tanto, al enfrentarnos al estudio de un sistema fluvial no se trata tanto de poder concluir la aplicabilidad del modelo, como a partir de éste u otros, entender cuál es su funcionamiento. Tal vez en ello resida su atractivo.

\section{AGRADECIMIENTOS}

A Narcís Prat por los comentarios y sugerencias que nos ha proporcionado durante la elaboración del manuscrito final.

\section{Bibliografía}

Agulló, M. (eds.), 1983. El agua en Madrid. 1. Diputación de Madrid.

American Public Health Association, 1985. Standard methods for the examination of water and waste water. АРНА. AWWH. WPCF.

Anderson, N.H. \& K.W. Cummins, 1979. The influence of diet on the life histories of aquatic insects. Journal of the Fisheries Research Board. Canada, 36: 335-342. 
Anderson, N.H. \& J.R. SEDELl, 1979. Detritus processing by macroinvertebrates in stream ecosystems. Ann. Rev. of Entomol., 24: 351-377.

BARMUTA, L.A., 1988. Benthic organic matter and macroinvertebrate functional feeding groups in a forested upland stream in Temperate Victoria. Verh. Internat. Verein. Limnol., 23: 1394-1398.

Barnes, J.R. \& G.W. Minshall (eds.), 1983. Stream ecology: Application and testing of general ecological theory. Plenum Press. New York and London. pp. 339.

BASS, D., 1982. The importance of a stratified design in sampling stream benthos. En: J.R. Davis (ed.) Proc. Symp. Recent Benthological Invest. in Texas and Adjacent States, Aquatic. Sci. Sect. Texas Academy of Sci. Austin. Texas.

Benke, A.C., C. Hall, C.P. Hawkins, R.H. Lowe-McConNEU J.A. Stanford, K. Suberkropp \& J.V. Ward, 1988. Bioenergetic consideration in the analysis of stream ecosystems. J.N. Am. Benthol. Soc. 7 (4): 480-502

CASADO, C., 1986. Composición y estructura de las comunidades de macroinvertebrados de un río intensamente regulado del sistema central: Río Lozoya (cuenca del Tajo). Tesis doctoral. Univ. Autónoma. Madrid.

Casado, C., D. García de Jalón, E. Barceló, M. Mayo \& O. SORIANO, 1987. Valoración ecológica de la red fluvial de la Comunidad de Madrid. Comunidad Autónoma de Madrid.

Casado, C., D. García de Jalón, C. Montes, E. Barceló $\&$ F. MENES, 1989. The effect of an irrigation and hydroelectric reservoir on its downstream communities. Regulated rivers: research and management, Vol. 4: 275-284.

Cummins, K.W., 1972. What is the river? Zoological description. En: R.T. OGlesby, C.A. CARlsson \& J.A. McCAnN (eds.) River Ecology and Man. Academic Press. 465 pp.

Cummins, K.W., 1973. Trophic relations of aquatic insects. Ann. Rev. entomol., 18: 183-206.

Cummins, K.W. \& M.J. KLug, 1979. Feeding ecology of stream invertebrates. Ann. Rev. Ecol. Syst. 10:147-172.

Cummins, K.W., J.R. Sedell, F.J. Swanson, G.W. Minshall, S.G. Fisher, C.E. Cushing, R.C. Petersen \& R.L. VANNOTE, 1982. Organic matter budgets for stream ecosystems: Problems in their evaluation. In: BARNES, J.R. \& G.M. Minshall (eds.), Stream ecology. Plenum Press. $399 \mathrm{pp}$

Cushing, C.E., C.D. McIntire, J.R. Sedell, K.W. Cummins, G.W. Minshall, R.C. Petersen \& R.L. Vannote, 1980. Comparative study of physical-chemical variables of stream using multivariate analysis. Arch. Hydrobiol., 89 (3): 343-352.

Czaya, E., 1983. Rivers of the world. Cambridge Univ. press. $246 \mathrm{pp}$.

Escarré, A., C. Garcia, F. Roda \& J. Terradas, 1984. Ecología del bosque esclerófilo mediterráneo. Investigación y ciencia, 95

GarCia de JALON, D., 1979. Estudio de las comunidades de macroinvertebrados, especialmente del orden tricóptera, del río Lozoya. Tesis Doctoral. Univ. Politécnica de Madrid.

García de JaLón, D. 1980. Efectos del embalse de «Pinilla» (Madrid) sobre las comunidades de macroinvertebrados bentónicos del río Lozoya. Bol. Est. Central de Ecología. ICONA, Vol. 9, n. ${ }^{\circ} 17:$ 47-52.
García de Jalón, D., C. Montes, E. Barceló, C. Casado \& F. MENES, 1988. Effects of hydroelectric escheme on fluvial ecosystems within the Spanish Pyrenees. Regulated Ri vers: Research \& Management, 2, 479-491.

GiUdicelli, J., M. DAKKI \& A. DiA, 1985. Caractéristiques abiotiques et hydrobiologiques des eaux courantes méditerranéennes. Verh. Internat. Verein. Limnol., 22: 2094-2101.

González, G., X. Millet, N. Prat, M.A. Puig, 1983. Patterns of macroinvertebrate distribution in the Llobregat river basins. N.E. (Spain). Verh. Intern. Ver. Limnol.: 2081-2087.

Hall, C.A.S., 1972. Migration and metabolism in a temperate stream ecosystem. Ecology, 53: 585-604.

HaWkins. C.P. \& J.R. SEDELL, 1981. Longitudinal and seasonal changes in functional organization of macroinvertebrate communities in four Oregon streams. Ecology, 62 (2): 387-397

Hughes, R.M. \& J.M. OMERMiK, 1983. An alternative for characterizing stream size. En: Fontaine (ed.), Dynamic of lotic ecosystems: 87-101.

LIND, O.T., 1974. Handbook of common methods in Limnology. Mosby. $199 \mathrm{pp}$

Margalef, R., 1984. Limnología. Omega. Barcelona.

MERRIT, R.W. \& K.W. CuMmINs (eds.), 1978. An introduction to the aquatic insects of North America. Kendall-Hunt, Dubuque, Lowa, USA.

Minshall, G.W., R.C. Petersen, C.W. Cummins, TH.L. Bott, J.R. Sedell, C.E. Cushing \& R.L. VANnote, 1983. Interbiome comparison of stream ecosystem dynamics. Eco logical monographs, 53 (1): 1-25.

Minshall, G.W., R.C. Petersen \& F. Curtis, 1985 b. Species richness in stream of different size from the same drai nage basin. Am. Nat., 125 (1): 16-38.

Minshall, G.W., K.W. Cummins, R.C. Petersen, C.E. Cus HING, D.A. BRuns, J.R. SEDELl \& R.L. VANnOTE, 1985 a Developments in stream ecosystem theory. Can. J. Fish. Aquat. Sci., 42: 1045-1055.

Montes, C., A. Llorca, A. Sterling, C. Casado, I. Cas Tro, A. Monzón, L. Santamaría \& D.G. DE JaLón, 1987. Directrices para la recuperación ecológica del tramo medio del río Manzanares. Canal de Isabel II. N. ${ }^{\circ} 18$.

Monzón, A., 1986. Organización longitudinal de comunidades de macroinvertebrados acuáticos en una cuenca del sistema central (Río Manzanares) y su relación con algunas variables funcionales. Grado de licenciatura. Univ. Autónoma. Madrid.

NEwBERN, L.A., 1978. Detritus transport in the New River, Virginia, M.S. Thesis, p. 40, Virginia Polytechnic Institute and State University, Blacksburg.

PRAT, N., 1981. The influence of reservoir discharge on benthic fauna in the river Ter, N.E. Spain. Series Entomologica, 20: 293-301.

Prat, N., M.A. Puig, G. González \& M.J. Tort, 1982. Pre dicció $i$ control de la qualitat de les aigües dels rius Besós Llobregat. Estudis i monografies $n .^{\circ} 6$. Servei del medi ambient. Barcelona.

Prat, N., M.A. Puig, G. González \& X. Millet, 1984. Chironomid longitudinal distribution and macroinvertebrates diversity along the Llobregat River (NE. Spain). Mem. Amer. Ent. Soc., 34: 267-278. 
Prat, N., G. González, I. Muñoz \& X. Millet, 1986. Community structure and colonization process in a mediterranean drainage basin. Proceeding 3rd European Congress of Entomology: 121-124.

Prat, N., I. Muñoz, J. Camp, F.A. Comin, J.R. Lucena, J. ROMERO \& M. VIDAL, 1988. Seasonal changes in particulate organic carbon and nitrogen in the river and drainage channels of the Ebro Delta (N.E. Spain). Verh. Internat. Verein. Limnol., 23: 1344-1349

Pringle, C.M., R.J. Naiman, G. Bretschio, J.R. Karr, H.W. Oswood, J.R. Webster, R.L. Welcomme \& M.J. Winterbourn, 1988. Patch dynamics in lotic systems: the stream as a mosaic. J.N. Am. Benthol. Soc. 7 (4): 513-524.

Puig, M.A., I. Bautista, M.J. Tort \& N. Prat, 1981. Les larves de tricoptères de la Rivière Llobregat (Catalogne, Espagne). Distribution longitudinale et regulation avec la qualité de l'eau. Series Entomologica, 20: 303-309.

RESH, V.H., 1979. Sampling variability and life history feature, basic considerations in the design of aquatic insect studies. J. Fish. Res, Board Can., 86: 290-311.

RESH, V.H. \& D.G. PRICE, 1984. Sequential sampling: a cost effective approach for monitoring benthic macroinvertebrates in environmental impact assessment. Environ. Manag.

Resh, V.H., A.V. Brown, A.P. Covich, M.E. GurzT, M.W. Li, G.W. Minshall, S.R. Reice, A.L. Sheldon, J.B. WALLACE, R.C. WISSMAR, 1988. The role of disturbance in stream ecology. J.N. Am. Benthol. Soc., 7 (4): 433-455.

Rigler, F.H. \& J.A. Downing, 1984. The calculation of secundary productivity. In: Downing \& Rigler (eds.), A manual on methos for the assessment of secundary productivity

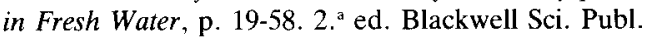

Robinson, C.T. \& G.W. Minshall, 1986. Effects of disturbance frequency on stream benthic community structure in relation to canopy cover and season. J.N. Am. Benthol. Soc. , 5: 237-248.

RYDER, G.I. \& D. SCOTT, 1988. The applicability of the River Continuum Concept to New Zealand streams. Verh. Internat. Verein. Limnol., 23: 1441-1445.
Sedell, J.R., R.J. Naiman, K.W. Cummins, G.W. Minshall \& R.L. VANNOTE, 1978. Transport of particulate organic material in streams as a function of physical processes. Int Verein. Theor. Angew. Limnol., 20: 1366-1375.

Schwennenker, B., R.A. Hellenthal, 1984. Sampling considerations in using stream insects for monitoring water quality. Envirn. Entomol., 13: 741-750.

StatzNer, B. \& B. Higler, 1985. Questions and comments on the river continuum concept. Com. Journ. Fish. aquat. Sci., 42 (5): 1038-1044.

STrahler, A.N., 1957. Quantitative analysis of watershed Geomorphology. Trans. Am. Geophys. Union, 38: 913-920.

Swain, F.M. \& R.W. MEader, 1958. Bottom sediments of southern part of Pyramid Lake, Nevada. J. Sedim. Petro$\log y, 28: 286-297$.

Vannote, R.L., G.W. Minshall, K.W. Cummins, J.R. SeDELL \& C. CUSHING, 1980. The river continuum concept. Can. J. Fish. Aquat. S., 37: 130-137.

VIDAL-ABARCA, M.R., 1985. Las aguas superficiales de la cuenca del río Segura (S.E. de España). Caracterización físico-química en relación al medio físico y humano. Tesis Doctoral. Univ. de Murcia.

VOLLENWEIDER, R.A., 1969. A manual on methods for measuring primary production in aquatic Environments. I. BP Handbook n. ${ }^{\circ}$ 12. Blackwell Oxford.

Wallace, J.B., D.H. Ross \& J.L. MEYER, 1982. Seston and dissolved organic carbon dynamics in a southern Appalachian stream: longitudinal changes in particle size composition. Ecology 63: 824-838.

WARD, J.V. \& J.A. STANDORD, 1983. The intermediate disturbance hypothesis: An explanation for biotic diversity pattern in lotic ecosystems. In: T.D. Fontaine \& S.M. Bartell (Edit.), Dynamics of lotic ecosystems. Ann. Arbor Science.

WARD, J.V. \& J.A. STANFORD, 1984. The serial discontinuity concept of lotic ecosystems. In: Fontaine, T.D. \& Bartell S.M. (eds.), Dynamics of lotic ecosystem. Ann. Arbor. Sci.

WETZEL, R.G. \& G.E. LinkENs, 1979. Limnological analyses. Saunder, Toronto.

Apéndice.- Categorías tróficas asignadas a los distintos grupos taxonómicos encontrados en el río Manzanares.

Trophic range of the different taxonomic groups presents in Manzanares stream.

$\mathrm{D}=$ depredadores, $\mathrm{Ra}=$ raspadores, $\mathrm{F}=$ filtradores, $\mathrm{Re}=$ recolectores, $\mathrm{De}=$ desmenuzadores. $(1)$ ejemplares capturados en estadio larvario exclusivamente.

\begin{tabular}{ll}
\hline Taxon & $\begin{array}{c}\text { Grupo } \\
\text { funcional }\end{array}$ \\
\hline TRICLADIDA & \\
F. Dugesiidae & \\
Dugesia sp & $\mathrm{D}$ \\
F. Planariidae & \\
$\quad$ Polycelis felina (Dalyell) & $\mathrm{D}$ \\
NEMATODA & $\mathrm{D}$ \\
GORDIACEA & $\mathrm{D}$ \\
GASTROPODA & \\
F. Ancylidae & \\
$\quad$ Ancylus fluviatilis Müll. & $\mathrm{Ra}$ \\
LAMELLIBRANCHIATA & \\
F. Sphaeriidae & \\
Pisidium sp. & $\mathrm{F}$ \\
OLIGOCHAETA & $\mathrm{Re}$
\end{tabular}

\begin{tabular}{ll}
\hline Taxon & $\begin{array}{c}\text { Grupo } \\
\text { funcional }\end{array}$ \\
\hline HIRUDINEA & \\
F. Glossiphoniidae & \\
Helobdella stagnalis (L.) & $\mathrm{D}$ \\
Batracobdella paludosa (Carena) & $\mathrm{D}$ \\
F. Erpobdellidae & \\
Erpobdella monoestriata (Gedr.) & $\mathrm{D}$ \\
Erpobdella sp. & $\mathrm{D}$ \\
INSECTA & \\
O. Ephemeropthera & \\
F. Baetidae & \\
Baetis gr. alpinus Pict. & $\mathrm{Re}$ \\
Baetis fuscatus L. & $\operatorname{Re}$ \\
Baetis muticus L. & $\operatorname{Re}$ \\
Baetis rhodani Pict. & $\operatorname{Re}$
\end{tabular}




\begin{tabular}{|c|c|c|c|}
\hline Taxon & $\begin{array}{c}\text { Grupo } \\
\text { funcional }\end{array}$ & Taxon & $\begin{array}{c}\text { Grupo } \\
\text { funcional }\end{array}$ \\
\hline F. Heptageniidae & & Limnius perrisis carinatus Perez & De \\
\hline Epeorus torrentium Etn. & $\mathrm{Ra}$ & Limnius sp. (1) & De \\
\hline Rhithrogena gr. hybrida Etn. & $\mathrm{Ra}$ & Oulimnius mayor Rey & De \\
\hline Ecdyonurus sp. & $\mathrm{Ra}$ & Oulimnius rivularis Rosenhauser & $\mathrm{De}$ \\
\hline F. Ephemerellidae & & O. Trichoptera & \\
\hline Serratela hispanica Etn. & $\mathrm{Ra}$ & F. Rhyacophilidae & \\
\hline F. Caenidae & & Rhyacophila meridionalis Ed. Pict & $\mathrm{D}$ \\
\hline Caenis luctuosa Burm. & $\mathrm{Re}$ & Rhyacophila munda McL. & $\mathrm{D}$ \\
\hline F. Leptophlebiidae & & Rhyacophila occidentalis McL. & $\mathrm{D}$ \\
\hline Calliarcys humilis Etn. & $\mathrm{Re}$ & Rhyacophila relicta $\mathbf{M c L}$. & $\mathrm{D}$ \\
\hline Habroleptoides sp. & $\mathrm{Re}$ & Rhyacophila sp. & $\mathrm{D}$ \\
\hline Habrophlebia fusca Curt. & $\operatorname{Re}$ & F. Glossosomatidae & \\
\hline O. Plecoptera & & Glossosoma privatum $\mathrm{McL}$. & $\mathrm{Ra}$ \\
\hline F. Taeniopterygidae & & Glossosoma sp. & $\mathrm{Ra}$ \\
\hline Brachyptera arcuata Klp. & $\mathrm{Ra}$ & Agapetus sp & Ra \\
\hline Brachyptera auberri Consgl. & $\mathrm{Ra}$ & F. Hydropsychidae & \\
\hline Brachyprera vera Bert y G. Tanago & $\mathrm{Ra}$ & Hydropsyche ambigua Schmid & $\mathrm{F}$ \\
\hline Brachyptera sp. & $\mathrm{Ra}$ & Hydropsyche dinarica Marinkoric & F \\
\hline F. Nemouridae & & Hydropsyche pellucida Curt. & $\mathrm{F}$ \\
\hline Amphinemum sp & $\mathrm{D}$ & Hydropsyche siltalai Dohler & $\mathrm{F}$ \\
\hline Nemoura $\mathrm{sp}$ & De & Hydropsyche tibialis $\mathrm{Mcl}$. & $F$ \\
\hline Protonemura meyer Pict. & De & Hydropsyche sp. & $\mathrm{F}$ \\
\hline F. Leuctridae & & F. Psychomyidae & \\
\hline Leuctra $\mathrm{sp}$ & $\mathrm{De}$ & Psychornya sp. & $\mathrm{Re}$ \\
\hline F. Capniidae & & F. Brachycentridae & \\
\hline Capnia bifrons Newm. & $\mathrm{De}$ & Micrasema longulum $\mathrm{Mcl}$ & $\mathrm{Ra}$ \\
\hline Capnioneura mitis Despax & $\mathrm{De}$ & Micrasema gr. minimum McL. & $\mathrm{Ra}$ \\
\hline F. Perlodidae & & F. Limnephilidae & \\
\hline Guadalgemus franzi & $\mathrm{D}$ & Limnephilus bipunctatus Curt & De \\
\hline Perlodes sp & $\mathrm{D}$ & Limnephilus rhombicus L. & De \\
\hline Isoperla $\mathrm{sp}$ & $\mathrm{D}$ & Potamophylax latipennis Curt & De \\
\hline F. Perlidae & & Stenophylax sp. & $\mathrm{De}$ \\
\hline Perla marginata $\mathrm{Pz}$ & $\mathrm{D}$ & Allogamus ligonifer McL. & $\mathrm{De}$ \\
\hline F. Chloroperlidae & & Allogamus sp. & $\mathrm{De}$ \\
\hline Siphonoperla torrentium Pict. & $\mathrm{D}$ & Stelophylicinae & $\mathrm{De}$ \\
\hline O. Odonata & & F. Goeridae & \\
\hline F. Calopterygidae & & Larcasia partita Navas & Ra \\
\hline Agrion splendens Harris & $\mathrm{D}$ & F. Lcpidostomatidac & \\
\hline F. Coenagrionidae & $\mathrm{D}$ & Lepidostoma hirtum Fbr. & $\mathrm{De}$ \\
\hline F. Gomphidae & & F. Sericostomatidae & De \\
\hline Onychogomphus uncatus (Chorr. 1840) & $\mathrm{D}$ & O. Diptera & \\
\hline O. Coleoptera & & F. Blephariceridae & $\mathrm{Ra}$ \\
\hline F. Gyrinidae & & F. Tipulidae & $\mathrm{D}$ \\
\hline Orectochillus villosus Müll. (1) & $\mathrm{D}$ & F. Limoniidae & $\mathrm{D}$ \\
\hline F. Hydraenidae & & F. Psychodidae & $\mathrm{Ra}$ \\
\hline Hydraena sharpi Rey & $\mathrm{De}$ & F. Simulidae & \\
\hline Hydraena testacea Curtis & $\mathrm{De}$ & Prosimulium sp. & $\mathrm{F}$ \\
\hline Hydraena sp. 1 & De & Simulium $\mathrm{sp}$ & $\mathrm{F}$ \\
\hline Hydraena sp. 2 & $\mathrm{De}$ & F. Chironomidae & \\
\hline Hydraena sp. 3 & $\mathrm{De}$ & Tanypodinae & $\mathrm{D}$ \\
\hline Hydraena sp. 4 & $\mathrm{De}$ & Orthoclidiinae & $\mathrm{Ra}$ \\
\hline F. Elmidae & & Tanytarsini & $\mathrm{F}$ \\
\hline Dupophilus brevis Mulsant \& Rey & $\mathrm{De}$ & F. Ceratopogonidae & $\mathrm{D}$ \\
\hline Elmis aenea Pn. Müll. & $\mathrm{De}$ & F. Empididae & $\mathrm{D}$ \\
\hline Elmis sp. (1) & $\mathrm{De}$ & F. Tabanidae & $\mathrm{D}$ \\
\hline Esolus angustatus $\mathrm{Ph}$. Müll. & De & F. Athericidae & $\mathrm{D}$ \\
\hline Esolus sp. (1) & De & & \\
\hline
\end{tabular}

